\title{
Wie viele Controller gibt es in Deutschland?
}

Rund 100.000 Beschäftigte können deutschlandweit dem beruflichen Rollenbild „Controller“ zugeordnet werden. Diese Zahl wurde erstmals vom Institut für Management und Controlling (IMC) an der WHU - Otto Beisheim School of Management ermittelt. Hierzu werteten Professor Utz Schäffer, Professor Jürgen Weber und Stefan Grunwald-Delitz die Arbeitsmarktdaten der Bundesagentur für Arbeit aus, die im Rahmen des Meldeverfahrens zur Sozialversicherung erhoben werden. Die Gesamtzahl setzt sich zusammen aus den direkt als Controller gemeldeten Mitarbeitern (69.428), Kostenrechnern (21.448) sowie einem geschätzten Anteil von Leitern im Bereich Rechnungswesen / Controlling / Revision (25.895). Die Zahlen gelten für den Stichtag 30.06.2013 und beziehen sich auf alle sozialversicherungspflichtig Beschäftigten. Der Analyse zufolge zeichnen sich direkt als Controller gemeldete Beschäftigte durch eine sehr gute Schul- sowie Berufsbildung aus. 71 Pro- zent besitzen das Abitur bzw. ein Fachabitur, 47 Prozent einen Diplom- bzw. Master-Abschluss. Zudem üben vornehmlich junge Arbeitnehmer den Controller-Beruf aus. 37 Prozent sind zwischen 30 und 39 Jahren alt, weniger als 50 Prozent sind 40 Jahre oder älter. Nach Auffassung des IMCs untermauern diese Zahlen die bereits weitverbreitete Ansicht, dass der Controller-Beruf häufig ein Einstieg für die Übernahme späterer Management-Positionen in Unternehmen ist.

Mehr zum Thema:

Parment, A.: Arbeitsmarkt und Karriere, in: Parment, A.: Die Generation Y, 2. Aufl., Wiesbaden 2013, S. 57 - 75, SSipip $^{*}$ www.springerprofessional.de/4714184

Vera Treitschke, Wiesbaden

\section{Deutsche Unternehmen sind Vertrauensweltmeister}

Mittelstand und Familienunternehmen sind die wesentlichen Vertrauenstreiber der deutschen Wirtschaft. So lautet eines der zentralen Ergebnisse des Edelman Trust Barometers 2014. Edelman Deutschland befragte 33.000 Menschen in 27 Ländern zu Vertrauen in und Glaubwürdigkeit von Regierungsund Nichtregierungsorganisationen, Wirtschaft und Medien. 77 Prozent gaben danach an, kleinen und mittleren Unternehmen zu vertrauen. Dagegen halten nur 49 Prozent Konzerne für glaubwürdig, lediglich 39 Prozent vertrauen börsennotierten Unternehmen. Am größten ist das Vertrauen mit 86 Prozent in Familienunternehmen. Insgesamt stieg der Vertrauenswert in die deutsche Wirtschaft gegenüber dem Vorjahr um neun Prozentpunkte auf 57 Prozent. Das ist der beste Wert seit Beginn der Erhebung im Jahr 2001.

Wichtig für das Vertrauen in ein Unternehmen ist der Erhebung zufolge der Unternehmenssitz. Deutsche Unternehmen profitieren von dem guten Ansehen Deutschlands in Bezug auf Vertrauen und Glaubwürdigkeit. Weltweit bringen ihnen die Befragten am meisten Vertrauen entgegen (80 Prozent), gefolgt von Unternehmen in Schweden und in der Schweiz (je 79 Prozent).

Soziale Verantwortung schafft weiteres Vertrauen in Unternehmen. Treiber sind laut der Erhebung vor allem soziale Aspekte wie der Umgang mit Mitarbeitern sowie die Kommunikation gegenüber Öffentlichkeit und Kunden. Dem Chief Executive Officer (CEO) kommt hier eine zentrale Rolle zu. „Der CEO der Zukunft wird zum Chief Engagement Officer“, so Edelman Deutschland.

Mehr zum Thema:

Vollmar, J./Becker, R./Hoffend, I. (Hrsg.): Macht des Vertrauens - Perspektiven und aktuelle Herausforderungen im unternehmerischen Kontext, Wiesbaden 2013,

SSIP] $^{*}$ www.springerprofessional.de/3723392

Vera Treitschke, Wiesbaden 